\title{
Mujeres, Violencia e Igualdad: Una cuestión de Derechos Humanos ${ }^{1}$
}

\author{
Women's rights in the Mexican state, from a human rights perspective for gender equality
}

Deniss Karina, González-Lozano²; Yahaira Berenice, Martínez-Pérez ${ }^{3}$

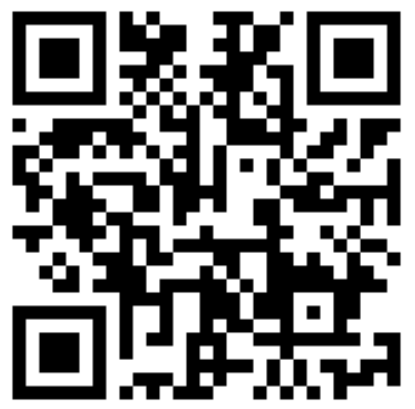

Fecha de recibido: $19-12-2020$

Fecha de aceptado: 28-02-2021

\section{(cc) BY-NC-ND}

Esta obra está bajo una licencia de Creative Commons Reconocimiento-NoComercial-

SinObraDerivada 4.0 Internacional

\begin{abstract}
RESUMEN
El presente trabajo es un producto de revisión documental que tuvo por objetivo analizar los derechos de las mujeres en el Estado mexicano desde una perspectiva de los derechos humanos para la igualdad de género. El estudio enfoca un análisis reflexivo a la norma jurídica desde un abordaje crítico jurídico. Se aplicó una metodología cualitativa desde el método exegético y dogmático partiendo del análisis documental. En el estudio se observa el marco teórico-jurídico de los derechos de las mujeres, así como la igualdad de género en el Estado Mexicano. En el estudio se observó que uno de los grupos que ha sufrido las graves consecuencias de la discriminación y de los cuales se han vulnerado sus derechos de manera arrasadora son las mujeres. Se concluye la necesidad de generar mecanismos de atención en perspectiva de derechos humanos para salvaguardar los derechos de las mujeres.
\end{abstract}

Palabras claves: Derechos de las mujeres, derechos humanos, violencia contra la mujer, igualdad de género.

\begin{abstract}
This work is a product of a documentary review that aimed to analyze the rights of women in the Mexican State from a human rights perspective for gender equality. The study focuses a reflective analysis of the legal norm from a critical legal approach. A qualitative methodology was applied from the exegetical and dogmatic method starting from the documentary analysis. The study observes the theoretical-legal framework of women's rights, as well as gender equality in the Mexican State. The study observed that one of the groups that have suffered the serious consequences of discrimination and whose rights have been violated in a devastating way are women. The need to generate attention mechanisms from a human rights perspective to safeguard women's rights is concluded.
\end{abstract}

Keywords: Gender equality, human rights, violence against women, women's rights.

Cómo referenciar este artículo:

González-Lozano., D., K., \& Martínez-Pérez., Y., B. (2021). Mujeres, Violencia e Igualdad: Una cuestión de Derechos Humanos. Revista Politica Globalidad y Ciudadanía, 7(14), 103-125. https://doi.org/10.29105/pgc7.14-6

\footnotetext{
${ }^{1}$ Este artículo es derivado del proyecto de investigación "Proceso de atención restaurativo en casos de violencia contra la mujer", cuenta con financiamiento de la Universidad Autónoma de Nuevo León.

${ }^{2}$ Doctora en Métodos Alternos de Solución de Conflictos por la Universidad Autónoma de Nuevo León, Monterrey. Docente de postgrado en Universidad Autónoma de Nuevo León. Email: dgonzalezlz@uanl.edu.mx; deniss_karina_g@hotmail.com. Orcid: https://orcid.org/0000-0003-3630-8501

${ }^{3}$ Doctora en Métodos Alternos y Solución de Conflictos por la Universidad Autónoma de Nuevo León, Monterrey. Profesor investigador de la Universidad Autónoma de Nuevo León. E-mail: yahairamtz@ hotmail.com; ymartinezp@uanl.edu.mx. Orcid: https://orcid.org/0000-0002-4047-7298
} 


\section{1.- INTRODUCCIÓN}

En el presente artículo se establece un análisis de los derechos de la mujer en el estado mexicano desde la perspectiva de los derechos humanos para la igualdad de género. En este sentido, se plantea la necesidad de describir los derechos de las mujeres desde el enfoque garantista de los estándares internacionales y locales, a través de la metodología cualitativa desde la observación descriptiva y el método exegético. Por consiguiente, se advierte el corpus iuris en igualdad de género.

Las garantías de las que todos los seres humanos gozamos son conocidas con el nombre de Derechos Humanos. Estos son inherentes desde nuestro nacimiento, sin importar raza, edad, posición económica o religión; son universales y deben ser respetados por todos los seres humanos.

Estos derechos del hombre y las mujeres también reciben esta denominación por corresponder a los seres humanos por su propia naturaleza y se consideran fundamentales e innatos (De Pina Vara, 2004), esto quiere decir, que nadie puede ser arrebatados de ninguno de ellos, pero si pueden padecer la violación de ellos en el trascurso de su vida.

La importancia de conocer el surgimiento y desarrollo de los Derechos Humanos a lo largo de la historia nos permite ubicar el esfuerzo que como humanidad hemos aplicado en para que hoy en día se puedan disfrutar, y a su vez seguir en la lucha para hacer valer todos y cada uno de nuestros derechos.

Las Naciones Unidas, a través de diversos instrumentos internacionales han reconocido a la mujer como vulnerable en las sociedades; dicho organismo destaca la Convención sobre la Eliminación de Todas las Formas de Discriminación contra la Mujer conocida por sus siglas CEDAW y la Convención Interamericana para Prevenir, Sancionar y Erradicar la Violencia contra las Mujeres denominada Convención de Belém Do Pará, entre algunas por mencionar.

Si bien las leyes adjetivas sustentan la salvaguarda de los derechos de las mujeres, existen datos que demuestran la constante violación a los derechos humanos que esta comunidad minoritaria padece (CNDI, 2018). Por lo tanto, se establece la necesidad de generar mecanismos de atención en perspectiva de derechos humanos para salvaguardar los derechos de las mujeres. 


\section{2.-FUNDAMENTO TEÓRICO}

Una visión general sobre derechos humanos.

La importancia de los derechos humanos ha sido palpada por la sociedad y los organismos internacionales desde tiempos remotos, es por ello por lo que al hablar del origen de los derechos humanos se deben mencionar algunos de sus antecedentes con la finalidad de comprender su concepto en la actualidad. Cabe referir los primeros antecedentes en sistemas jurídicos, éstos se remontan desde el Derecho Romano y el Derecho Canónico, mismos que se posicionaron en Europa Continental y América Latina a partir del siglo XIII (Daza Gómez, Torres, \& Barritia, 2006).

Como ante sala, los Derechos Humanos marcan sus orígenes en el año 539 a. de C. cuando Ciro el Grande, con la conquista de Babilonia, liberó a los esclavos, declarando que toda persona era libre de escoger su religión sin ser perseguidos y estableció la igualdad racial. Estos decretos fueron grabados en un cilindro de barro cocido, conocido como Cilindro de Ciro, documento que se reconoce como el primer escrito de los derechos humanos del mundo (ONU, Organización de las Naciones Unidas, 2012).

Por su parte la escuela estoica basándose en estos decretos ampliaron el concepto al hablar de una ley natural universal a la que se adhería todo hombre por el uso de su razón, partiendo de una concepción del cosmos como un todo ordenado, el humano ante su capacidad de raciocinio se ubica en un lugar único por su facultad de comprender la estructura, la unidad del todo y conocer así su papel dentro de ella. Destacando que al obrar con el todo gracias a la razón, el ser humano encuentra la felicidad y con ello el valor de la justicia (Guerrero Reyes, 2017).

Si bien la filosofía estoica abre la puerta con la idea de la razón universal, por su parte, el cristianismo bajo su concepción espiritual sobre la idea de fraternidad humana amplia la idea de lo que es "justo“ cómo orden objetivo ante su concepción de la búsqueda de justicia (López Moreno \& López Arellano, 2015), otorgando al ser humano un valor superior, promulgando la igualdad por ser todos hijos de Dios logrando con ello el rechazo a todo acto de esclavitud para dar paso al establecimiento de valores morales tanto a nivel colectivo como del individuo mismo.

Bajo esta perspectiva moral se destaca las contribuciones de Santo Tomás de Aquino al asentar que de existir un conflicto entre lo social y lo individual en relación con lo material, era necesario y justo que prevaleciera el bien común. Así, al mantener una buena conducta por medio de los hábitos 
beneficiosos para la persona y para los que lo rodean, se logra mantener la armonía del mundo, es decir, se logra así una virtud moral que consiste en la constante y firme voluntad de dar a Dios y al prójimo lo que le es debido y justo, sobre poniendo así el derecho humano (Yon B., 2005).

Con el avance de la sociedad hace su aparición otro punto clave en la historia de los Derechos Humanos, el Rey de Inglaterra en el año de 1215 firma el primer documento que reconoce los derechos de las personas, declarando en él, que nadie podría invalidarlos logrando con ello que los derechos de las personas fueron reconocidos y protegidos de los que estaban en el poder. Dicho documento es conocido como La Carta Magna, considerada como uno de los documentos legales más importantes por compilar de forma escrita, el conjunto de normas y principios que garantizaba el efectivo cumplimiento de los derechos humanos dentro de toda sociedad y convirtiéndolo el documento que marca el inicio de la democracia moderna (Unidos por los Derechos Humanos, 2019).

A partir del acontecimiento antes mencionado una oleada de importantes momentos se destacan cuando se habla del desarrollo de los Derechos Humanos como: La Petición de los Derechos en 1628 como la Declaración de Libertades Civiles, La Declaración de la Independencia de Estados Unidos en 1776 que proclamaba el derecho a la vida, la libertad y la búsqueda de la felicidad, La Declaración de los Derechos del Hombre y el Ciudadano en 1789 en la época de la Revolución Francesa en el cual este documento de Francia establecía que todos los ciudadanos son iguales ante la ley, todos estos eventos enfocados con un solo fin, el reconocimiento de la igualdad entre los seres humanos.

Ante todos los movimientos que se suscitaban tanto en Estados Unidos como en Europa, en 1864 se lleva a cabo la primera Convención de Ginebra para promover el cuidado de los heridos de guerra, en donde dieciséis países europeos y varios países de América se congregaron con el propósito de adoptar un convenio para el tratamiento de los soldados heridos en combate, en donde se obligaba a proveer atención médica sin discriminación a personal militar herido o enfermo y de respetar el trasponte y el equipo del personal médico con el signo distintivo de la cruz roja sobre fondo blanco (Herrero de la Fuente, 2014).

Es así como la historia permite ver que estos acuerdos marcaron la ruptura radical con las formas de poder que se tenía sobre los pueblos, comenzando por establecerse la igualdad de derechos considerados ineludibles a la naturaleza del hombre, y dando como resultado las primeras Constituciones modernas estableciendo el modelo de democracia representativa, el gobierno de las leyes, la posibilidad 
de la alternancia en el poder y la administración de justicia en base a principios establecidos legalmente en el parlamento. Sin duda daba comienzo una nueva era de la sociedad occidental que con sus cambios permearía de manera profunda al mundo entero.

La gran cantidad de afectados a consecuencia de los conflictos armados y la devastación que dejaba a su paso el término de la Segunda Guerra Mundial impulsa a los Estados alrededor del mundo a fundar en 1945 lo que hoy en día se conoce como la Organización de las Naciones Unidas -ONU-. Tras los estragos ocasionados por dicho acontecimiento y la preocupación de todas las naciones participantes generó en la comunidad internacional una inminente necesidad por plasmar en blanco y negro una carta de derechos que afirmara los valores defendidos en la lucha contra el fascismo y el nazismo, así nace la Declaración Universal de los Derechos Humanos (DUDH), proclamados por la ONU en 1948.

En este documento (DUDH) que al paso de los años se ha ratificado, que se reconoce y es aceptado internacionalmente se presentan al mundo los 30 artículos que declaran los derechos inherentes a todos los seres humanos con la finalidad de promover, proteger y conseguir: libertad, justicia y paz para todos por igual.

Lo antes mencionado se deja asentado con el artículo 1 de dicha declaración que a la letra dice (ONU, 2019): “ Todos los seres humanos nacen libres e iguales en dignidad y derechos y, dotados como están de razón y conciencia, deben comportarse fraternalmente los unos con los otros. “ dejando claro la intención de prevalencia de los valores humanos como la bondad, la empatía, generosidad, responsabilidad, entre algunos por mencionar.

Para reforzar lo antes mencionado dicha Declaración dentro de su articulado va de derechos más generales a más específicos y se acogen otros derechos personales y sociales más concretos, que se relacionan con la interacción de los individuos que conviven y trabajan en sociedad como ejemplo el Artículo 12 el cual menciona “Nadie será objeto de injerencias arbitrarias en su vida privada, su familia, su domicilio o su correspondencia, ni de ataques a su honra o a su reputación. Toda persona tiene derecho a la protección de la ley contra tales injerencias o ataques.

Bajo esta misma Declaración de Derechos, la lista se extiende con la finalidad de abarcar aspectos relacionados a los derechos de pensamiento, conciencia, religión o libertad política, como ejemplo el artículo 18 de dicha Declaración que dice: 'Toda persona tiene derecho a la libertad de pensamiento, de conciencia y de religión; este derecho incluye la libertad de cambiar de religión o de creencia, así como 
González-Lozano., D., K. \& Martínez-Pérez., Y., B.

la libertad de manifestar su religión o su creencia, individual y colectivamente, [...] ." o bien el artículo 21 que manifiesta que: "1. Toda persona tiene derecho a participar en el gobierno de su país, directamente o por medio de representantes libremente escogidos. Es así como se puede apreciar la intención de cuidar todos los aspectos de la vida de los individuos buscando con ello una convivencia pacífica y armoniosa entre las personas y extenderlo hasta las naciones del mundo.

Los últimos artículos de la Declaración de Derechos Humanos se enfocan en derechos que abarcan derechos relacionados con las condiciones de vida, educación, la cultura, ciencia y la protección de las personas ante cualquier discriminación ante dicho documento. Si bien es verdad que los derechos humanos establecidos dentro de la DUDH son universales e inherentes a las personas, es de reconocer que no siempre se alcanzan a completar a lo largo del mundo entero, y que las naciones siguen esforzándose por alcanzar o preservar al menos los más esenciales, dejando una tarea continua para los dirigentes de los países en la búsqueda de una vida más justa e igualitaria para todos los habitantes del planeta.

A partir de este acontecimiento, se destaca el Pacto Internacional de Derechos Civiles y Políticos y el Pacto Internacional de Derechos Económicos Sociales y Culturales, organizaciones como la Organización de Estados Americanos - OEA, tratados y convenciones enfocadas a trabajar en favor de los grupos más vulnerables resaltando la Convención para la Eliminación de la Violencia contra la Mujer, entre otros.

Ahora bien, al hablar de Derechos Humanos no solo es importante destacar los momentos claves que han marcado su curso durante la evolución humana, es necesario a su vez establecer la conceptualización cuando se habla de ellos. El buen entendimiento sobre el concepto de los Derechos Humanos permite establecer un marco de referencia ante un concepto que, si bien se considera universal, deja una tarea importante para todos aquellos que se dedican al estudio de estos, ya que es difícil encontrar una unanimidad en cuando a su definición.

Una de las definiciones básicas cuando se habla de los Derechos Humanos es la proporcionada por la Organización de las Naciones Unidas la cual expresa que los Derechos Humanos (...) Son derechos inherentes a todos los seres humanos, sin distinción alguna de raza, sexo, nacionalidad, origen étnico, lengua, religión o cualquier otra condición (...) (ONU, 2012), es decir, corresponden a todas las personas, sin discriminación alguna. 
Bajo este orden de ideas y ante el amplio cúmulo de definiciones del término, Peces-Barba citado por (González Valencia, 2006) hace su aportación definiendo a los derechos humanos como:

La facultad que la norma atribuye de protección de la persona, en lo referente a la vida, a su libertad, a su participación política y social, o a cualquier otro aspecto fundamental que afecte a su desarrollo integral como persona, en una comunidad de hombres libres, exigiendo el respeto de los demás hombres, de los grupos sociales y del Estado en caso de infracción.

Otros autores como Castán Tobeñas señala que los Derechos Humanos se pueden definir como:

Aquellos derechos fundamentales de la persona humana -considerada en su aspecto individual como comunitario- que corresponde a éste por razón de su propia naturaleza (de esencia, a un mismo tiempo, corpórea, espiritual y social), y que deben ser reconocidos y respetados por todo poder y autoridad, y toda noma jurídica positiva, cediendo, no obstante, en su ejercicio ante las exigencias del bien común (Castán Tobeñas, 1976).

En esta misma perspectiva hace sus aportaciones Burgoa al afirmar que "los derechos se traducen en imperativos éticos emanados de la naturaleza del hombre que se traduce en el respeto a su vida, dignidad y libertad, en su dimensión de persona” (Burgoa Orihuela, 1995).

Es así como se destaca en ellos un mismo fin al momento de definir y conceptualizar los Derechos Humanos recalcando que su esencia es la protección y respeto de los aspectos fundamentales que permitan el desarrollo integral de una persona, emanando de su condición de su naturaleza humana.

Sin embargo para autores como Pérez Luño (1984) pone énfasis en la necesidad de hacer una división entre los derechos humanos como: “Un conjunto de facultades e instituciones que, en cada momento histórico, concretan las exigencias de la dignidad, la libertad y la igualdad humanas, las cuales deben ser reconocidas positivamente por los ordenamientos jurídicos a nivel nacional e internacional" refiriéndose a que estos son los que posee una persona y que por ende el Estado debe respetar sin distinción ni discriminación alguna, y los derechos fundamentales como: “Aquellos derechos humanos garantizados por el ordenamiento jurídico positivo, en la mayor parte de los casos en su normativa constitucional, y que suelen gozar de una tutela reforzada" dándole el énfasis a que estos son aquellas facultades que posee una persona y que son reconocidos a través de un ordenamiento jurídico vigente, es decir, son reconocidos legalmente y protegidos a través de garantías procesales. 
González-Lozano., D., K. \& Martínez-Pérez., Y., B.

Siguiendo este mismo orden de ideas, ONU Mujeres ha manifestado su interés de la participación de las mujeres para dirimir conflictos a través de procesos de negociación y conciliación, en busca de sensibilizar el desarrollo en los procesos de mediación con enfoque de género, bajo este mismo contexto, algunos autores reconocen la aplicación de los programas de justicia restaurativa con enfoque a las garantías internacionales de los derechos humanos (Sauceda Villeda \& Martínez Pérez, 2018).

Si bien no se puede decir que existe una decisión unánime ante una definición universal de los Derechos Humanos, se puede destacar que los autores concuerdan en que los Derechos Humanos revisten características que los singularizan y que son necesarios para comprender su importancia.

En este mismo orden de ideas, según Carpizo (2011) las características de los Derechos Humanos son:

Universalidad: significa que todo ser humano posee una serie de derechos con independencia del país en que haya nacido o habite.

Historicidad: se refiere a tres aspectos diversos: a) La evolución de la civilización; b) Nuevos problemas, necesidades y retos, y c) El contexto social y cultural de cada país.

Progresividad: implica que su concepción y protección nacional, regional e internacional se va ampliando irreversiblemente, tanto en lo que se refiere al número y contenido de ellos como a la eficacia de su control.

Aspecto protector: se ampara a toda persona humana, incluso se ha llegado a sostener que los derechos humanos no deben proteger sólo a la persona, sino también a la comunidad nacional.

Indivisibilidad: implica que todos los derechos forman una unidad.

Eficacia directa: significa que los derechos humanos reconocidos en la Constitución y en los instrumentos internacionales ratificados por un país, vinculan obligatoriamente a todos los poderes públicos -Ejecutivo, Legislativo, Judicial y órganos constitucionales autónomos-, así como a autoridades, grupos y personas, y para ello, no es necesario que una ley desarrolle los alcances de ese derecho humano, aún en el supuesto de que la Constitución señale la existencia de esa ley.

Por su parte, el multicitado organismo internacional reconoce los derechos humanos como universales e inalienables, es decir, son universales por el simple hecho de ser persona, pues todos los ciudadanos gozamos de ellos. Son inalienables ya que no pueden renunciarse o cederse. Menciona que los derechos humanos son indivisibles e interdependientes por lo que se encuentran estrechamente 
relacionados, pero ninguno de ellos es más importante que otro y a su vez, ningún derecho tiene prioridad sobre el otro.

Ahora bien, equitativo y no discriminativo, al referirse que todas las personas al nacer gozamos del derecho de igualdad y de la dignidad de los derechos, así como la no discriminación, misma que se contempla mediante la resolución 2106-A, en la Convención Internacional sobre la Eliminación de todas las Formas de Discriminación Racial y la Convención sobre la Eliminación de todas las Formas de Discriminación contra la Mujeres (Naciones Unidas, 1965). Por lo tanto, se advierte que los derechos de las mujeres son iguales ante las normas jurídicas por lo que reconoce salvaguardar el respeto a la dignidad de todas las personas refiriéndonos a los que se integran en poblaciones minoritarias (Martínez Pérez, Sauceda Villeda, \& Moreno Rodríguez, 2020).

Contexto jurídico internacional y nacional de los derechos de las mujeres.

Uno de los temas que hoy en día toma los escaparates tanto de organizaciones, gobiernos y sociedad son aquellos que están relacionados con las mujeres. Y como no ha de ser así, si es visiblemente la desventaja que existe hacia ellas en todos los ámbitos de nuestra vida cotidiana. Se advierte la diferencia que se da entre hombres y mujeres actualmente alrededor del mundo, que son constantes los esfuerzos por buscar la manera de estrechar los privilegios que preexisten entre ellos.

Estos esfuerzos se ven reflejados en el ámbito de los Derechos Humanos, ya que la igualdad entre las mujeres y los hombres, así como lograr la eliminación de todas las formas de discriminación contra la mujer forma parte de los derechos humanos fundamentales y sobre todo del interés del máximo organismo enfocado en mantener la paz, la seguridad del mundo y defender los derechos humanos: las Naciones Unidas.

Como se ha venido mencionado en párrafos anteriores, desde la fundación de las Naciones Unidas, se ha buscado hacer hincapié en promulgar, proteger y promover la igualdad tanto de hombres como mujeres. Esto se puede ver reflejado en La Carta de las Naciones Unidas en 1945 que establece dentro de sus objetivos el interés por "reafirmar la fe en los derechos fundamentales del hombre, en la dignidad y el valor de la persona humana y en la igualdad de derechos de hombres y mujeres" (Naciones Unidas, 2019). 
González-Lozano., D., K. \& Martínez-Pérez., Y., B.

Así mismo, en el ordinal primero se reafirma dicho propósito al manifestar como principio” (...) el respeto a los derechos humanos y a las libertades fundamentales de todos, sin hacer distinción por motivos de raza, sexo, idioma o religión (...)” (Naciones Unidas, 2019). Po lo que se ha de destacar que durante la creación de la Declaración Universal de Derechos Humanos en 1948 se dio un arduo debate acerca del uso del término "todos los hombres" por el uso de un término más neutro en cuanto al género, y fue así como se incorporó en su redacción el término "todos los seres humanos" y “todas las personas" dejando con ello en claro que dicho documento hace referencia tanto a hombres como mujeres por igual. (Naciones Unidas, 2014).

En este parámetro cabe destacar diversos instrumentos internacionales de especial relevancia en la violencia contra la mujer, como la Convención de los Derechos Políticos de la Mujer (1953), la Convención sobre la Nacionalidad de la Mujer Casada (1957), la Convención sobre el consentimiento para el matrimonio (1962), la Convención Internacional sobre la Eliminación de todas las Formas de Discriminación Racial y la Convención sobre la Eliminación de todas las Formas de Discriminación contra la Mujeres (1965), El Pacto Internacional de los Derechos Civiles y Políticos (1966), el Pacto de San José de Costa Rica (1969), la Convención sobre la eliminación de todas las formas de discriminación contra la mujer (CEDAW) (1979), la Convención Interamericana para Prevenir, Sancionar y Erradicar la Violencia contra la Mujer (Convención de Belém do Pará) (1994), las Estrategias y Medidas Prácticas Modelo de las Naciones Unidas para Eliminar la Violencia contra los Niños en el ámbito de la Prevención del Delito y la Justicia Penal (2014), sin dejar de mencionar, La Declaración Universal de los Derechos Humanos (1948).

Aún y en el entendido de que la Declaración Universal de los Derechos Humanos, generaliza los sexos, cabe mencionar que destaca su universalidad e inclusión para todos los seres humanos, por lo que permite su validación como instrumento para los derechos humanos de las mujeres. El reconocimiento frente una necesidad de proteger a las mujeres como grupo vulnerable y víctimas de una sociedad que daba el privilegio a los hombres, la Asamblea General de las Naciones Unidas solicita a la Comisión de la Condición Jurídica y Social de la Mujer la elaboración de una Declaración sobre la eliminación de la discriminación contra la mujer, la cual fue aprobada en 1967.

Ante una realidad mundial sobre estrategias que favorecieran el hacer valer y promover los derechos universales de las mujeres, en 1979, nace el instrumento más importante sobre los derechos de las mujeres jurídicamente vinculante, nos referimos a la Convención sobre la Eliminación de Todas las 
Formas de Discriminación contra la Mujer-CEDAW- el cual con el paso de los años en 1981 entra en vigor como tratado internacional.

Dicha Convención forma el parteaguas de la defensa de los Derechos de las Mujeres en el mundo, ya que es el instrumento más completo y sólido que promueve sus derechos y recoge toda la normativa precedente respecto a los derechos de las mujeres en sus diversos ámbitos como legales, culturales, reproductivos y económicos, por mencionar algunos.

En una breve descripción, la CEDAW consta de un preámbulo y 30 artículos. La fuerza que dicho instrumento representa para los derechos de las mujeres se aprecia desde su inicio, en donde en su primera parte da una definición clara y concisa sobre lo que significa "la discriminación contra la mujer", y que a la letra dice en su artículo 1 (CEDAW, 1979):

...toda distinción, exclusión o restricción basada en el sexo que tenga por objeto o por resultado menoscabar o anular el reconocimiento, goce o ejercicio por la mujer, independientemente de su estado civil, sobre la base de la igualdad del hombre y la mujer, de los derechos humanos y las libertades fundamentales en las esferas política, económica, social, cultural y civil o en cualquier otra esfera.

El articulado que se extiende dentro de dicha Convención en esta primera parte compromete a los Estados Partes a adoptar políticas y medidas legislativas para eliminar todas las formas de discriminación y con ello asegurar el pleno desarrollo y adelanto de la mujer.

En su artículo 5, Los Estados Partes se comprometen a tomar medidas apropiadas para la modificación de los patrones socioculturales de conductas que contribuyan y perpetúen la idea de inferioridad o superioridad de cualquiera de los sexos y así mismo garantizar la educación familiar bajo la comprensión de la maternidad como función social y dándole reconocimiento a la responsabilidad compartida de los hijos a ambos padres.

En su segunda parte, la Convención se enfoca en su articulado del 7 al 9 en tomar todas las medidas apropiadas para la eliminación de la discriminación contra la mujer en cuanto a sus derechos civiles y políticos, dejando una referencia clara en cuanto a las condiciones de igualdad ante el voto, ocupación de cargo públicos, participación en organizaciones y asociaciones no gubernamentales, la oportunidad de representar a su gobierno en el plano internacional y todo lo relacionado con la adquisición, cambio o conservación de su nacionalidad. 
La tercera parte de dicha Convención hacer referencia en sus artículos del 10 al 14, a los derechos de las mujeres en el campo socioeconómico, educación y formación, al trabajo y maternidad, a la salud, al crédito y a las condiciones de las mujeres en las zonas rurales. La parte final que va desde su artículo 15 al 30 hace referencia a los mecanismos de aplicación por parte de la Convención para examinar y asegurar el completo ejercicio de lo acordado dentro de ella.

Uno de los mecanismos adoptados para velar por la eficiencia y eficacia de dicha Convención, fue el establecimiento del Comité para la Eliminación de la Discriminación contra la Mujer, órgano encargado de vigilar su aplicación, hacer el estudio de los informes proporcionados por los Estados Miembros y devolverle a cada Estado Parte el resultado de su análisis con las recomendaciones inmediatas a ejecutar para enriquecer las medidas encaminadas a erradicar la discriminación en la vida de las mujeres.

Dicho comité establece directrices en la presentación de informes específicos que en su conjunto constituyen estándares armonizados para la presentación de informes de conformidad con la Convención sobre la eliminación de todas las formas de discriminación contra la mujer (Naciones Unidas, 2009). De esta manera, en el año 2018 México rindió su noveno informe ante la CEDAW manifestando los avances en las reformas legislativas y los progresos para erradicar la violencia de género que se ha vivido en nuestro país ante los feminicidios, con ello salvaguardar los derechos de las mujeres (Naciones Unidas, 2018). Sin embargo, plantea la carencia de mecanismos eficaces en la promoción de la igualdad de género, así como las constantes vulneraciones a los actos discriminatorios por motivos de sexo que viven las mujeres.

Ahora bien, bajo esta misma idea acerca del reconocimiento de los derechos de las mujeres en el mundo, se deben resaltar acontecimientos que contribuyeron para que hoy en día se continué la lucha sobre los derechos universales de las mujeres. Con la finalidad de impulsar la importancia de las mujeres en el mundo, en 1975 se declara el Año Internacional de la Mujer, con la finalidad de llamar la atención sobre la igualdad entre mujeres y hombres y destacar su contribución al desarrollo y la paz del mundo.

Ese mismo año se celebra en la Ciudad de México, la Primera Conferencia Mundial sobre la Mujer y años más tarde se da lugar a conferencias mundiales como la de Copenhague en 1980 y la de Nairobi en 1985. Sin embargo, no fue hasta 1993 cuando se logra elevar las cuestiones de género a la 
categoría de temas trasversales y se dejó de ver las problemáticas de las mujeres en el mundo como asuntos independientes de los países.

A partir de aquí se da un giro a la visión de la defensa de los derechos de la mujer, dejando plasmada su importancia en la Declaración sobre la eliminación de la violencia contra la mujer, al ser reconocida la violencia como una problemática social grave, logrando la compresión internacional de que esta problemática constituye una violación de sus derechos humanos y a su vez una forma de discriminación.

Por último pero no menos importante otra de las plataformas que destaca en importancia al proteger los derechos universales de las mujeres a nivel internacional es la Organización de los Estados Americanos -OEA- la cual negoció la Convención Interamericana para Prevenir, Castigar y Erradicar la Violencia contra las Mujeres conocida como la Convención de Belém Do Pará (1994) en la cual se recalca el derecho de toda mujer a una vida libre de violencia, tanto en el ámbito público como en el privado.

Dicha Convención deja plasmados aquellos derechos que han de ser protegidos para todas las mujeres, los cuales se manifiestan en su artículo 4 que a la letra dice:

Toda mujer tiene derecho al reconocimiento, goce, ejercicio y protección de todos los derechos humanos y a las libertades consagradas por los instrumentos regionales e internacionales sobre derechos humanos. Estos derechos comprenden, entre otros:

a. el derecho a que se respete su vida;

b. el derecho a que se respete su integridad física, psíquica y moral;

c. el derecho a la libertad y a la seguridad personal;

d. el derecho a no ser sometida a torturas;

e. el derecho a que se respete la dignidad inherente a su persona y que se proteja a su familia;

f. el derecho a igualdad de protección ante la ley y de la ley;

g. el derecho a un recurso sencillo y rápido ante los tribunales competentes, que la ampare contra actos que violen sus derechos;

h. el derecho a libertad de asociación;

i. el derecho a la libertad de profesar la religión y las creencias propias dentro de la ley, y 
González-Lozano., D., K. \& Martínez-Pérez., Y., B.

j. el derecho a tener igualdad de acceso a las funciones públicas de su país y a participar en los asuntos públicos, incluyendo la toma de decisiones.

Bajo esta visión, se demuestra la preocupación universal de reconocer los derechos de las mujeres a la luz de los derechos humanos en perspectiva de género con la finalidad de poder dar un trato justo ante un mundo diseñado y que es manejado bajo la tutela de los hombres, pero que sin duda con el paso de los años ha logrado tener poco a poco una apertura hacia los grupos más vulnerables, en donde se ubica a las mujeres.

En atención al marco internacional, el estado mexicano ha externado su compromiso para erradicar la violencia contra la mujer y alcanzar la igualdad entre las mujeres y los hombres, por lo que integra un corpus iuris para salvaguardar los derechos de las mujeres, entre los cuales se destacan la Constitución Política de los Estados Unidos Mexicanos, la Ley para la Protección de los Derechos de Niñas, Niños y Adolescentes expedida en el año 2000, Ley General para la Igualdad entre mujeres y hombres (2006), la Ley General de Acceso de las Mujeres a una Vida Libre de Violencia (2007), el Reglamento de la Ley General de Acceso de las Mujeres a una Vida Libre de Violencia (2008), La Ley General de los Derechos de Niñas, Niños y Adolescentes (2014), La Ley Federal de Telecomunicaciones y Radiodifusión (2014), el Protocolo de actuación para quienes imparten justicia en asuntos que involucren a niñas, niños y adolescentes por la Suprema Corte de Justicia de la Nación (2014), la Ley General de Víctimas (2017), La Ley General para Prevenir, Investigar y Sancionar la Tortura y otros Tratos o Penas Crueles, Inhumanos o Degradantes, (2017), el Reglamento para el funcionamiento del Sistema Nacional para Prevenir, Atender, Sancionar y Erradicar la Violencia contra las Mujeres (2020), el Protocolo para Juzgar con Perspectiva de Género (2020).

En este mismo orden de ideas, nuestro sistema jurídico Constitucional contempla el imperio de Derecho en la legalidad de ajustar la aplicación en la norma jurídica mediante el artículo 133 bajo la siguiente tesitura:

"Esta Constitución, las leyes del Congreso de la Unión que emanen de ella y todos los tratados que estén de acuerdo con la misma, celebrados y que se celebren por el presidente de la República, con aprobación del Senado, serán la Ley Suprema de toda la Unión. Los jueces de cada entidad federativa se arreglarán a dicha Constitución, leyes y tratados, a pesar de las 
disposiciones en contrario que pueda haber en las Constituciones o leyes de las entidades federativas".

Por lo que, en el año 2011, se genera un paradigma de justicia con enfoque a los derechos humanos fortaleciendo los artículos $1^{\circ}, 15,18$, entre algunos otros por mencionar. Por su parte el artículo primero reconoce a todas las personas como inherentes a los derechos humanos, garantizando los principios rectores de universalidad, interdependencia, indivisibilidad y progresividad.

Hace especial énfasis en el párrafo quinto a la no discriminación e igualdad de género de la siguiente manera:

“Queda prohibida toda discriminación motivada por origen étnico o nacional, el género, la edad, las discapacidades, la condición social, las condiciones de salud, la religión, las opiniones, las preferencias sexuales, el estado civil o cualquier otra que atente contra la dignidad humana y tenga por objeto anular o menoscabar los derechos y libertades de las personas”.

Por su parte el ordinal 15 reconoce la no vulneración a los derechos humanos y a los instrumentos internacionales al referir que "No se autoriza la celebración de convenios o tratados en virtud de los que se alteren los derechos humanos reconocidos por esta Constitución y en los tratados internacionales de los que el Estado Mexicano sea parte”. Ahora bien, el párrafo sexto del artículo 18 reconoce la igualdad de condición de los derechos humanos en justicia para adolescentes, refiriéndose como tal a niños, niñas y jóvenes hombres y mujeres.

De esta manera, la Constitución de los Estados Unidos Mexicanos establece que todas las personas gozarán de los derechos humanos que están reconocidos en la referida Carta Magna y los tratados internacionales de los que el Estado Mexicano sea parte, dejando sustentado con esto que los derechos humanos de las mujeres se encuentran garantizados de manera universal en todo el territorio nacional (ONU Mujeres, 2018).

Queda manifiesto que, el respeto a los Derechos humanos no es de orden pasivo, toda vez que es obligación del Estado brindar a su población seguridad, paz y orden, así como implementar políticas públicas de enfoque de género que erradiquen, prevengan y mitiguen la violencia contra la mujer. 


\section{Aportaciones en la igualdad de género. Caso México.}

Una de las problemáticas más graves por ser considerada una forma de discriminación y violación a los derechos humanos de las mujeres versa en la violencia ejercida hacia la mujer tanto en el ámbito público como en lo privado, lo que la convierte en la piedra angular no solo a nivel mundial, sino también para cada país (González Lozano \& Sáenz López, 2018), que hace frente a ella, en donde México no es la excepción.

La violencia contra la mujer es una problemática social que no reconoce estrato social, religión, educación o etnia, misma que causa consecuencias no solo a la mujeres que la padecen sino a sus hijos e hijas, generando con ello que las afectaciones permeen y dejen secuelas para todos, visualizando desde la violencia en el noviazgo (García Hidalgo \& López Valdés, 2014; ONU, 2016; Navarro Góngora \& Pereira Miragaia, 2000), (Miranda-Medina, Martínez Pérez, \& Otros, 2019), (Sáenz López \& González Lozano, 2016)

Las organizaciones internacionales con el apoyo de sus diversas comisiones permiten apoyar a los Estados Partes a tener un aliado para el análisis y elaboración de sugerencias ante aquellas necesidades de mejora que cada uno como país debe realizar para mejorar la calidad de vida de sus ciudadanos, dando a su vez el tiempo y la soberanía para que se ejerzan los avances de acuerdo a las normas y leyes nacionales en vigor de cada pueblo.

La Encuesta Nacional sobre la Dinámica de las Relaciones en los Hogares (ENDIREH, 2016), y la Encuesta Nacional sobre Violencia contra las Mujeres (ENVIM, 2006) son herramientas que utiliza la Nación con la finalidad de evidenciar la violencia contra la mujer y cuantificarla para así poder tomar las medidas necesarias para la instrumentación de mecanismos que permitan prevenir, sancionar y erradicar el problema en todo el país.

Por su parte la ENDIREH 2016, deja como resultado que en México de las mujeres de 15 años y más, $66.1 \%$ han enfrentado al menos un incidente de violencia por parte de cualquier agresor, alguna vez en su vida, $43.9 \%$ de las mujeres han sufrido violencia por parte de su actual o última pareja, esposo o novio, a lo largo de su relación, y en relación a los espacios públicos o comunitarios, 34.3\% de las mujeres han experimentado algún tipo de violencia sexual, datos que de manera general son alarmantes. 
La ENVIM 2006 deja de manifiesto que en cuanto a la violencia contra la mujer el 33.3\% de las mujeres encuestadas señalan que han vivido violencia en el último año de relación, 35\% de las mujeres señalaron que han tenido más de una relación violenta y $60 \%$ de las mujeres encuestadas señalan que han tenido toda su vida.

Ambas encuestas ponen de manifiesto que la violencia contra las mujeres no es un fenómeno aislado, sino que afecta a un importante número de mujeres, promoviendo con sus análisis de datos la importancia como problemática de carácter público y así formar parte de las prioridades del gobierno como parte de su plan de seguridad nacional y dar sustento a desarrollo de las políticas públicas para la prevención y atención del tema.

Es importante hacer mención que aunado a los señalamientos recibidos por parte de organismos internacionales, esta problemática toma mayor importancia ya que casos particulares han llegado a la Corte Interamericana de Derechos Humanos - Corte IDH- y ha sentenciado al Estado Mexicano en referencia a violaciones a los derechos humanos de las mujeres en los casos de: González y otras (Campo Algodonero), caso Fernández Ortega y por último caso Rosendo Cantú (Gobierno de la República, 2014).

El riesgo que vive la mujer en México es alarmante en la actualidad ya que el cuidado de su seguridad ha dejado mucho que desear, situación que se confirma al encontrar los siguientes dato: 28 de sus 32 entidades federativas, que equivalen al $56 \%$ del territorio nacional, se encuentra formalmente en Alerta de Violencia de Género contra la Mujer, destacando dentro de los estados de mayor preocupación Veracruz, Guerrero, Jalisco, Morelos y el Estado de México (Observatorio Ciudadano Nacional del Feminicidio, 2019).

En este sentido, ante la prevalencia de la impunidad y la agudización de la violencia contra las mujeres en México, el OCNF hace un llamado al Gobierno Federal para:

El fortalecimiento de la Comisión Nacional para Prevenir y Erradicar la Violencia Contra las Mujeres (CONAVIM) con los recursos humanos y materiales idóneos para cumplir de manera efectiva la encomienda de su creación.

El desarrollo de mecanismos institucionales rectores a nivel federal que, de forma permanente y sistemática, realicen un verdadero ejercicio de planeación estratégica, monitoreo y evaluación de todas las AVG, y demás acciones que emprendan para garantizar y proteger los derechos de las mujeres en México. 
González-Lozano., D., K. \& Martínez-Pérez., Y., B.

Actuar de manera integral, desde una perspectiva nacional, que también recupere las realidades estatales y municipales, para que se implementen acciones dirigidas a las particularidades de dichas problemáticas.

Las instancias federales -particularmente la CONAVIM- se involucren y corresponsabilicen en cinco ejes estratégicos: Planear, Supervisar (monitorear y evaluar), Apoyar, Proponer y Coordinar las AVGM.

Los datos anteriores dejan de manifiesto como el Derecho a la No Violencia contra la mujer se ve poco protegido hoy en México y que la garantía de sus derechos no ha sido una prioridad de Estado, poniendo en peligro la integridad de niñas y mujeres a lo largo y ancho de todo el país, dejando a la luz la falta de atención a la causa raíz de la violencia contra las mujeres en toda la nación, la carencia de implementación de mecanismos que vayan dirigidos a dar seguimiento a las medidas aplicadas y por consecuencia colocar a la violencia contra las mujeres como prioridad para las medidas de Seguridad Nacional de México.

De lo anteriormente mencionado, se puede demostrar que el mismo estado mexicano ha sido señalado por la Corte Interamericana de Derechos Humanos por casos de violencia de género, cobra especial relevancia la sentencia conocida como González y otras "Campo Algodonero” (2019) y la sentencia emitida bajo el nombre "Mujeres víctimas de tortura sexual en Atenco" (2018).

Respecto al caso Campo Algodonero se manifiesta la responsabilidad el Estado por la desaparición y ulterior muerte de tres jóvenes mujeres (González, Herrera y Ramos), se señala al estado mexicano responsable ante la carencia de medidas de protección y prevención en crímenes de mujeres, así como la existencia de patrón en violencia de género dejando en evidencia centenares de muertes en Cd. Juárez ante la indebida diligencia en las investigaciones; de manera colateral el Caso Mujeres Víctimas de Tortura Sexual en Atenco Vs. México en perjuicio de Dios dada, Jiménez, Romero, Selvas, Rosales, Velasco, Cuevas, Méndez, Sánchez, Torres y Hernández Martínez ha determinado que las once mujeres detenidas fueron víctimas de actos de tortura, violencia física, sexual y psicológica, así como el incumplimiento característico del ius poenale del Estado ante estos hechos.

Tras estas reflexiones, México ha señalado su preocupación ante las problemáticas a las que se enfrentan las mujeres en lo referente al enfoque de género y la violación de sus derechos humanos, ya que impacta diferentes esferas de su vida, tanto en lo laboral, familiar, social, personal e institucional. 
Mediante informe ante la CEDAW (2018), plantea la persistencia de los patrones naturalizados en violencia de género, materializados en violencia física, sexual, económica y patrimonial; los estereotipos discriminatorios para definir responsabilidades entre mujeres y hombres; la escasa participación de la mujer en el ámbito público y privado; las constantes desapariciones forzadas que afectan a las mujeres, la falta de sensibilización por el Estado con las víctimas indirectas así como las obstaculizaciones en la aplicación a los mecanismos de alerta de género.

En este contexto, nos encontramos ante el desafío de fomentar la participación de las mujeres en la vida pública, de implementar estrategias para coadyuvar en la igualdad sustantiva y con ello superar los estereotipos discriminatorios que sufren las mujeres en familia y sociedad.

\section{3.-MÉTODO}

Diseño.

En este trabajo de investigación se planteó un método descriptivo de enfoque cualitativo (Hernandez Sampieri, Fernández Collado, \& Baptista Lucio, 2014), donde a través del método exegético se interpretan compilaciones legislativas locales, nacionales e internacionales en las siguientes categorías: 1) los derechos de las mujeres, 2) los derechos humanos y 3) la igualdad de género.

\section{Instrumentos.}

El instrumento parte desde el análisis documental de estándares internacionales y legislación mexicana, también se consultaron diversos textos bibliográficos y casos de sentencia en violencia contra la mujer. Bajo este contexto, se analizó una reflexión crítica jurídica de carácter argumentativo con la finalidad de indagar los derechos de las mujeres a la luz de los derechos humanos para la igualdad de género. Posteriormente se plantean resultados y conclusiones.

\section{Procedimiento.}

En este trabajo de investigación se utiliza un diseño de investigación cualitativa, mediante la cual se lleva a cabo la revisión de textos y normas desde un análisis documental, partiendo desde la reflexión en la interpretación de la norma jurídica. Ésta se argumenta con el diseño metodológico del marco teórico, mediante el estudio de los estándares nacionales e internacionales, característica fundamental de la 
González-Lozano., D., K. \& Martínez-Pérez., Y., B.

exégesis, sin dejar de mencionar diversas teorías relativas al fenómeno de estudio. Posteriormente se analizan los resultados y conclusiones.

\section{4.-RESULTADOS}

De primera instancia, cabe referir que los derechos de las mujeres se encuentran sustentados desde el máximo órgano internacional y nacional, bajo este rubro la Carta Magna refiere los derechos de las mujeres en igualdad a los derechos humanos, es decir enmarca universales e inalienables, indivisibles $e$ interdependientes equitativo y no discriminativo, por lo que se asume el derecho a la igualdad entre el hombre y la mujer.

Se demostró que la mayoría de los Estados Miembros de las Naciones Unidas, ratificaron a la CEDAW como un referente para definir los derechos humanos de las mujeres a nivel mundial, por lo que deja en evidencia que el instrumento más representativo para erradicar las formas de discriminación contra la mujer es la CEDAW, en él se promueve la igualdad de género mediante el acceso igualitario en los diferentes ámbitos políticos, sociales, culturales y económicos, señalando que los roles de cultura, así como los estereotipos influyen de manera negativa en actos discriminatorios y de violencia.

Asimismo, en la compilación legislativa encontramos la igualdad sustantiva, al exigir marco jurídico aplicable en el respeto y salvaguarda de los derechos de las mujeres para la igualdad de género, sin embargo, en las sentencias de Caso Campo Algodonero y Caso Mujeres Víctimas de Tortura Sexual en Atenco Vs. México se aduce la desigualdad de género en los ámbitos público y privado. De esta manera pone en acento la respuesta política por lo que se requiere implementar políticas públicas que integren mecanismos de atención con criterios de valoración en la igualdad y equidad entre las mujeres y hombres.

\section{5.-CONCLUSIONES}

El surgimiento de los Derechos Humanos garantiza los derechos fundamentales y las libertades que gozan todos los seres humanos, incluidas las mujeres. Se concluye que estos derechos deben estar protegidos por las leyes y la sociedad sin distinción de sexo, raza, estatus social, cultural o religión.

Sin embargo, hoy en día, las mujeres siguen sufriendo de discriminación y violencia tanto en el ámbito público y privado con cifras alarmantes al grado de ser una problemática de alto impacto para la sociedad expandida por toda la nación. 
Se identificó que el estado de emergencia que impera en México sobre la violencia de la que son víctimas las mujeres muestra la necesidad de dirigir el esfuerzo para generar instrumentos que permitan a los dirigentes del País el acertado desarrollo de políticas públicas enfocadas a garantizar la seguridad de las mujeres y a su vez profundizar en el conocimiento sobre el vínculo entre violencia contra las mujeres y la consecuencias de un pobre desarrollo humano derivado de la violación y discriminación de las que son víctimas.

Si bien, es de reconocer que en México se ha logrado un avance en la normatividad que regula y garantiza el acceso de las mujeres a una vida libre de violencia, también es necesario destacar que continúa palpable la necesidad inmediata de armonizar las leyes estatales con los instrumentos internacionales en materia de derechos humanos de las mujeres, así como mejorar la calidad del servicio de atención por parte de las autoridades para garantizar la protección de las víctimas de violencia.

De manera específica en México, la situación que viven las mujeres recalca la vulneración de los derechos humanos en igualdad de género por lo que debemos considerar que poner fin a la violencia contra las mujeres es pieza clave para el logro de la igualdad de género y su empoderamiento, y que como resultado permitiría un país genuinamente camino al desarrollo.

\section{REFERENCIAS}

Burgoa Orihuela, I. (1995). Las garantías individuales. México: Porrúa.

Carpizo, J. (2011). Los derechos humanos: naturaleza, denominación y características. Cuestiones Constitucionales. Revista Mexicana de Derecho Constitucional(25), 10-24.

Castán Tobeñas, J. (1976). Los derechos del hombre. Madrid: Reus.

CEDAW. (1979). Convención sobre la eliminación de todas las formas de eliminación de la violencia contra la mujer. www.un.org

CNDI. (2018). Análisis cuantitativo y cualitativo de las Violaciones de los Derechos Humanos de Grupos Vulnerables. Comisión Nacional de los Derechos Humanos. www.cndh.org.mx

Convención Belém Do Pará.

http://www.unicef.org/argentina/spanish/ar_insumos_ConvencionBelem.pdf

Corte Interamericana de Derechos Humanos. (28 de noviembre de 2018). Caso Mujeres víctimas de tortura sexual Atenco vs. México. https://www.corteidh.or.cr/docs/casos/articulos/seriec_371_esp.pdf

Corte Interamericana de Derechos Humanos. (16 de noviembre de 2019). Caso González y otras (Campo Algodonero) Vs México. https://www.corteidh.or.cr/docs/casos/articulos/seriec_205_esp.pdf

Daza Gómez, C., Torres, S. G., \& Barritia, C. E. (2006). Principios Generales del Juicio Oral Penal. México: Flores Editor. 
González-Lozano., D., K. \& Martínez-Pérez., Y., B.

De Pina Vara, R. (2004). Diccionario de Derecho (33 ed.). México: Porrúa.

ENDIREH. (2016). Encuensta Nacional sobre la Dinámica de las Relaciones en los Hogares. México: INEGI.

ENVIM. (2006). Encuesta Nacional sobre la Violencia contra las Mujeres. México.

García Hidalgo, L., \& López Valdés, D. C. (2014). Violencia contra la mujer adulta en las relaciones de pareja. MEDISA, 18(2), 181-187.

Gobierno de la República. (2014). Programa Integral para Prevenir, Atender, Sancionar y Erradicar la Violencia contra las Mujeres 2014-2018. México. http://americalatinagenera.org/newsite/includes/fichas/politica/MEXICO.pdf

González Lozano, D. K., \& Sáenz López, K. C. (2018). Análisis conceptual de la violencia contra la mujer en el ámbito privado y su inclusión en la Justicia Restaurativa. Pensamiento Americano, 11(20), 53-71.

González Valencia, A. (2006). La justicia social como fin primoridal de los Derechos Humanos. México: Universidad Juárez Autónoma de Tabasco.

Guerrero Reyes, O. E. (31 de Enero de 2017). La Solidaridad Humana, Algunos aportes desde la escuela estoica. (Tesis de maestria). 49 -52. Getafe, Madrid, España: Universidad Carlos III de Madrid.

Hernandez Sampieri, R., Fernández Collado, C., \& Baptista Lucio, P. (2014). Metodología de la Investigación. México: McGraw-Hill.

Herrero de la Fuente, A. A. (2014). En el Sequicentenario del Convenio de Ginebra de 1864. Revista de Estudios Europeos(64), 9-10.

López Moreno, S., \& López Arellano, O. (2015). Orígen y naturaleza de los derechos humanos. En O. López Arellano, \& S. López Moreno, Derecho a la Salud en México (págs. 17- 49). México: Casa abierta al tiempo.

Martínez Pérez, Y. B., Sauceda Villeda, B. J., \& Moreno Rodríguez, M. S. (2020). Los Derechos Procesales en grupos vulnerables desde la normativa nacional e internacional. Revista Política, Globalidad y Ciudadanía, 6(12), 225-245.

Miranda-Medina, C. F., Martínez Pérez, Y. B., \& Otros. (2019). Percepción sobre la violencia en las relaciones amorosas de una Facultad de Derecho y Criminología en una Universidad del Norte de México.Erg@omnes Revista jurídica,11(1), 171-195.

Naciones Unidas. (1948). Declaración Universal de los Derechos Humanos. https://www.un.org/es/universal-declaration-human-rights/

Naciones Unidas. (1965). Convención Internacional sobre la Eliminación de todas las Formas de Discriminación Racial. https://www.ohchr.org/sp/professionalinterest/pages/cerd.aspx

Naciones Unidas. (3 de junio de 2009). Comité para la eliminación de la discriminación contrta la mujer. (Instrumentos Internacionales de Herechos Humanos) https://tbinternet.ohchr.org/_layouts/15/treatybodyexternal/Download.aspx?symbolno=HRI\%20 GEN\%202\%20Rev.6\&Lang=en

Naciones Unidas. (2014). Los derechos de la mujer son derechos humanos. Nueva York y Ginebra: Naciones Unidas. 
Naciones Unidas. (2018). ONU Mujeres. (México ante la CEDAW). https://www.onu.org.mx/wpcontent/uploads/2019/04/MEXICO-ANTE-LA-CEDAW-2018-web.pdf

Naciones Unidas. (2019). Naciones Unidas. https://www.un.org/es/carta-de-las-nacionesunidas/index.html

Navarro Góngora, J., \& Pereira Miragaia, J. (2000). Parejas en situaciones especiales. Barcelona: Paidós.

Observatorio Ciudadano Nacional del Feminicidio. (15 de Enero de 2019). Comunicado de prensa. México. https://issuu.com/cencos/docs/190115_comunicado_exige_ocnf_una_co

ONU. (2012). Organización de las Naciones Unidas. Recuperado el 4 de 03 de 2019, de Departamento de Información Publica de las Naciones Unidas, Declaración Universal de los Derechos Humanos: http://www.un.org/es/documents/udhr/history.shtml.

ONU. (Enero de 2016). Organización de las Naciones Unidas. http://www.who.int/mediacentre/factsheets/fs239/es/

ONU. (2019). Naciones Unidas. http://www.un.org/es/universal-declaration-human-rights/

ONU Mujeres. (2018). Del compromiso a la acción. http://americalatinagenera.org/newsite/includes/fichas/fichas/spanish/MEXICO.pdf

Pérez Luño, A. (1984). Derechos Humanos, Estado de Derecho y Constitución. Madrid: Tecnos.

Sáenz López, K. C., \& González Lozano, D. K. (2016). Desarrollo de la justicia restaurativa en el ámbito de la violencia doméstica. Revsita de la Facultad de Derecho(40), 245-260.

Sauceda Villeda, B. J., \& Martínez Pérez, Y. B. (2018). Los MASC desde el Sistema para el Desarrollo Integral de la Familia en México. Revista de la Facultad de Derecho, 1-31.

Unidos por los Derechos Humanos. (2019). Unidos por los derechos humanos. https://www.unidosporlosderechoshumanos.mx/what-are-human-rights/brief-history/

Yon B., L. (2005). La justicia de acuerdo a Santo Tomás de Aquino. Eleuteria(3), 1-6. 